\title{
Prediction of the Impact of Deleterious Nonsynonymous Single Nucleotide Polymorphisms on the Human RRM2B Gene: A Molecular Modeling Study
}

\author{
Chaimaa Ait El Cadi $\mathbb{D}^{1,2}$ Al Mehdi Krami ${ }^{1 D},{ }^{1}$ Hicham Charoute ${ }^{(D)},{ }^{1}$ Zouhair Elkarhat, ${ }^{1}$ \\ Najat Sifeddine, ${ }^{1}$ Hamid Lakhiari, ${ }^{2}$ Hassan Rouba, ${ }^{1}$ Abdelhamid Barakat, ${ }^{1}$ \\ and Halima Nahili $\mathbb{D}^{1}$ \\ ${ }^{1}$ Laboratory of Genomics and Human Genetics, Institut Pasteur du Maroc, 20360 Casablanca, Morocco \\ ${ }^{2}$ Laboratory of Biosciences, Functional Integrated and Molecular Exploration-LBEFIM-, Biology Department, Faculty of Sciences and \\ Technics of Mohammedia, University of Hassan II, Mohammedia 28806, Morocco
}

Correspondence should be addressed to Halima Nahili; halima.nahili@pasteur.ma

Received 29 April 2020; Revised 4 July 2020; Accepted 6 July 2020; Published 26 July 2020

Academic Editor: Maxim P. Evstigneev

Copyright ( $) 2020$ Chaimaa Ait El Cadi et al. This is an open access article distributed under the Creative Commons Attribution License, which permits unrestricted use, distribution, and reproduction in any medium, provided the original work is properly cited.

\begin{abstract}
$R R M 2 B$ gene encodes ribonucleoside-diphosphate reductase subunit M2 B, the p53-inducible small subunit (p53R2) of ribonucleotide reductase (RNR), an enzyme catalyzing dNTP synthesis for mitochondrial DNA. Defects in this gene may cause severe mitochondrial disease affecting mainly the nervous system. This study is aimed at examining the effect of deleterious nonsynonymous SNP (nsSNP) on the structure of the RRM2B protein, using a variety of prediction tools followed by a molecular modeling analysis. After using 13 algorithms, 19 nsSNPs were predicted deleterious. Among these variants, 18 decreased the protein stability and 16 were localized in very highly conserved regions. Protein 3D structure analysis showed that 18 variants changed amino acid interactions. These results concur with what has been found in experimental trials; 7 deleterious nsSNPs were previously reported in patients suffering from genetic disorders affecting the nervous system. Thus, our study will provide useful information to design more efficient and fast genetic tests to find $R R M 2 B$ gene mutations.
\end{abstract}

\section{Introduction}

Mitochondria are semiautonomous, self-reproducing organelles that occur in the cytoplasm of all cells of most eukaryotes. In that manner, a mitochondrial function can be provided by either mitochondrial or nuclear DNA; therefore, any disruption of both genetic materials can lead to severe mitochondrial diseases [1]. Human ribonucleotide reductase (RNR) is a heterotetramer enzyme that catalyzes the synthesis of dNTPs by direct reduction of ribonucleoside diphosphates to deoxyribonucleoside diphosphates required for DNA replication; it consists of two subunits: a large catalytic subunit called R1 and a small subunit called R2 [2,3]. Actually, cells have two forms of the R2 subunit, a form regulated by the cell cycle expressed abundantly at the $\mathrm{S}$ phase and a second form regulated by the $\mathrm{p} 53$ tumor suppressor protein called p53R2.

This form is necessary for the synthesis and repair of mitochondrial DNA (mtDNA) inside nonproliferative cells $[3,4]$. Indeed, this form is encoded by a gene known as ribonucleoside diphosphate reductase subunit $\mathrm{M} 2 \mathrm{~B}$ or $R R M 2 B$ located on chromosome 8 in position $8 q 22.3$ [5].

Mutations within the $R R M 2 B$ gene result in a variety of inherited mitochondrial diseases either recessive or dominant and can be divided into 2 major types: diseases characterized by mtDNA depletion and diseases characterized by multiple deletions of the mtDNA. mtDNA depletion, where inheritance is autosomal recessive, is generally characterized by severe multisystemic manifestations such as encephalomyopathy with proximal renal tubulopathy usually fatal in 


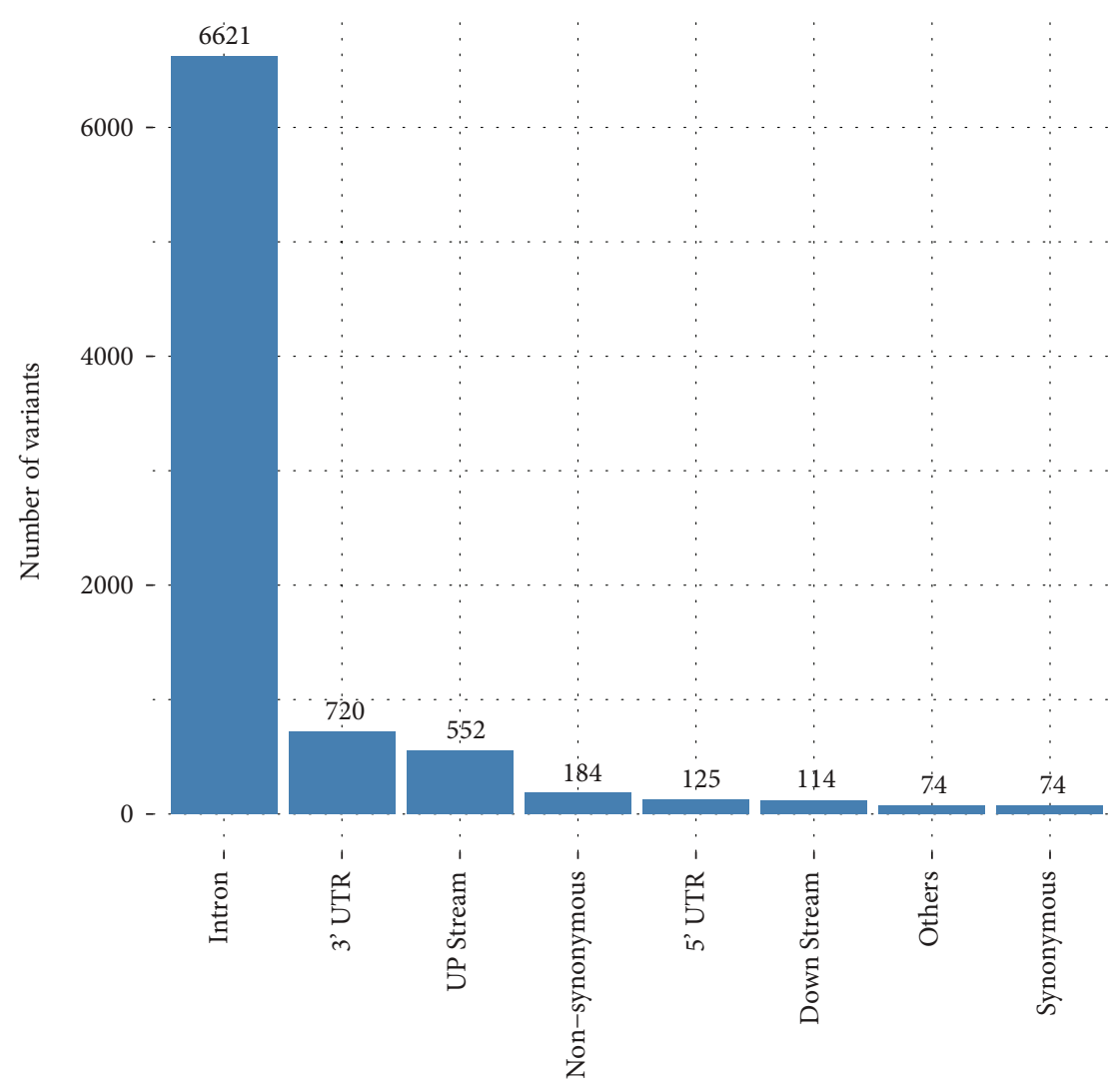

Type of variants

FIgURE 1: Distribution of SNPs present in the RRM2B gene.

early age [5-10]. However, multiple mtDNA deletions cause tissue-specific cytochrome $c$ oxidase (COX) deficiency. Inheritance can be either autosomal recessive with progressive external ophthalmoplegia (PEO) and multisystem involvement manifesting during early childhood/adulthood or autosomal dominant with less severe manifestations appearing during late adulthood [11-15]. We can also mention Kearns-Sayre syndrome (KSS) as one of the rare phenotypes that may occur due to mutations in the $R R M 2 B$ gene. $R R M 2 B$ gene variants can be found within all exons, but exon 9 is specifically considered as a mutation hotspot, since variations in this region are responsible for a truncated protein presumed to cause a dominant-negative or gain-of-function effect on the heterotetrameric structure of the RNR enzyme [11, 12].

There is currently a large number of in silico tools used to predict the structural impact caused by amino acid (aa) changes in a given molecule. These tools are not very accurate; however, they can still be used as an initial filter of potentially deleterious changes. Subsequently, we can first resort to molecular modeling which allows us to observe the impact of these changes on the protein $3 \mathrm{D}$ structure. Then, advanced analyses such as molecular dynamics simulation can be used to better assess the impact caused by these variants. However, the use of such a method requires a large computing power.
In this study, we will investigate the impact of nonsynonymous single nucleotide polymorphisms (nsSNPs) on the $R R M 2 B$ protein, followed by molecular modeling analysis. This will allow us to evaluate the effect of potentially deleterious variations on the protein structure.

\section{Data and Methods}

2.1. Data and Sequence Extraction. The sequence of the $R R M 2 B$ protein has been extracted in Fasta format from UniProt (ID: Q7LG56) (https://www.uniprot.org/uniprot/ Q7LG56). Meanwhile, the SNPs were generated from NCBI's dbSNP database (https://www.ncbi.nlm.nih.gov/ $\operatorname{snp} /)$.

2.2. Most Damaging Variant Identification. In order to have an idea about the impact of nsSNPs on the structure of the $R R M 2 B$ protein, we used the following algorithms: SIFT [16], POLYPHEN [17], SNAP [18], PhD-SNP [19], Condel [20], PROVEAN [21], M-Cap [22], LRT [23], Mutation Assessor [24], Mutation Taster [25], PredictSNP [26], MAPP [27], and PANTHER [28]. Similarly to other studies [29], we maintained the standard cutoff or the threshold scores of all prediction tools to select the most deleterious amino acid changes on the protein function. 


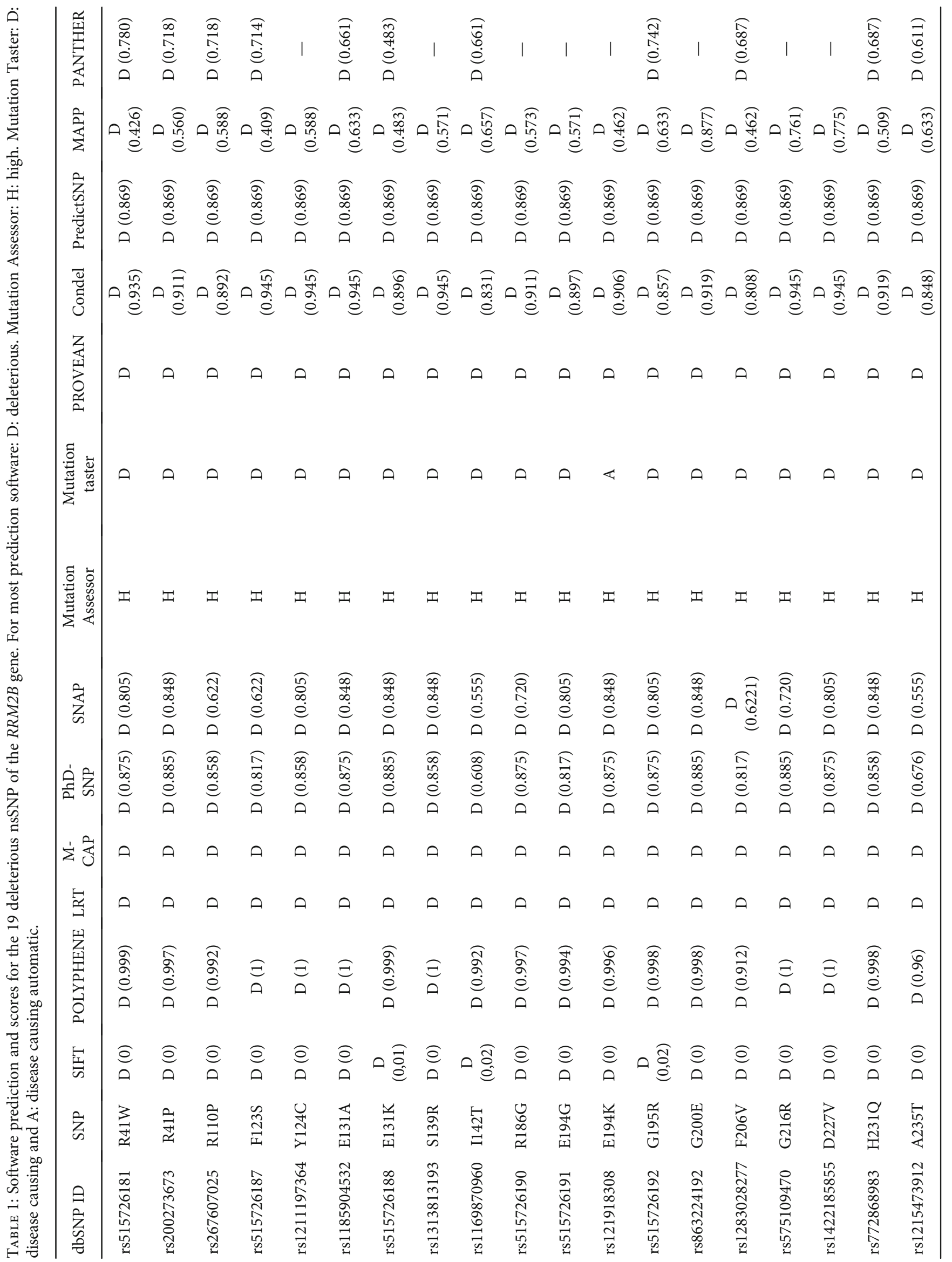


TABLE 2: Stability software predictions and DDG scores for the 19 deleterious nsSNPs of the RRM2B gene.

\begin{tabular}{|c|c|c|c|c|c|}
\hline \multirow{2}{*}{ dbSNP ID } & \multirow{2}{*}{ SNP } & \multicolumn{2}{|c|}{ I-Mutant } & \multicolumn{2}{|c|}{ Mupro } \\
\hline & & Prediction & DDG value & Prediction & DDG value \\
\hline rs515726181 & $\mathrm{R} 41 \mathrm{~W}$ & Decrease & -0.57 & Decrease & -1.809 \\
\hline rs200273673 & R41P & Decrease & -1.06 & Decrease & -2.19 \\
\hline rs267607025 & R110P & Decrease & -1.47 & Decrease & -1.312 \\
\hline rs515726187 & F123S & Decrease & -2.49 & Decrease & -1.688 \\
\hline rs1211197364 & $\mathrm{Y} 124 \mathrm{C}$ & Increase & 0.56 & Decrease & -0.935 \\
\hline rs1185904532 & E131A & Decrease & -0.04 & Decrease & -1.24 \\
\hline rs515726188 & $\mathrm{E} 131 \mathrm{~K}$ & Decrease & -0.63 & Decrease & -1.644 \\
\hline rs1313813193 & S139R & Decrease & -0.75 & Decrease & -0.849 \\
\hline rs1169870960 & $\mathrm{I} 142 \mathrm{~T}$ & Decrease & -2.42 & Decrease & -3.633 \\
\hline rs515726190 & R186G & Decrease & -1.91 & Decrease & -1.33 \\
\hline rs515726191 & E194G & Decrease & -2.61 & Decrease & -1.121 \\
\hline rs121918308 & E194K & Decrease & -1.5 & Decrease & -0.523 \\
\hline rs515726192 & G195R & Decrease & -0.82 & Decrease & -0.393 \\
\hline rs863224192 & G200E & Decrease & -0.94 & Decrease & -0.399 \\
\hline rs1283028277 & F206V & Decrease & -2.2 & Decrease & -1.157 \\
\hline rs575109470 & G216R & Decrease & -1.08 & Decrease & -0.503 \\
\hline rs1422185855 & $\mathrm{D} 227 \mathrm{~V}$ & Decrease & -0.92 & Decrease & -0.507 \\
\hline rs7728688983 & H231Q & Decrease & -0.62 & Decrease & -0.199 \\
\hline rs1215473912 & $\mathrm{A} 235 \mathrm{~T}$ & Decrease & -1.63 & Decrease & -1.261 \\
\hline
\end{tabular}

2.3. Stability and Conservation Analysis. I-Mutant (http:// folding.biofold.org/i-mutant/) is a stability prediction software that measures the degree of protein destabilization and gives the predicted free energy change value DDG, which is the Gibbs free energy value from the mutated protein minus the Gibbs free energy value from the wild type expressed in $\mathrm{Kcal} / \mathrm{mol}$. The software judges an SNP "Increasing" the stability of the protein if the DDG value is superior to 0 or "Decreasing" it if the DDG value is below 0 .

Mupro (http://mupro.proteomics.ics.uci.edu/) is also another stability prediction web server that uses support vector machines and neural networks, to predict the impact of a single site amino acid mutation on protein stability. This tool provides a prediction reliability score between -1 and 1 .

Conservation analysis was conducted by ConSurf (http:// consurf.tau.ac.il/), a web server identifying the functional regions of a protein by evaluating the degree of its conservation.

We also attempted to examine the exact number of conserved residues upon the $R R M 2 B$ protein sequence, using the Structurally Conserved Region (SCR) prediction web server (http://prodata.swmed.edu/scr_prediction/index.php).

2.4. Molecular Modeling of Native and Variant Forms of the $R R M 2 B$ Protein. For the purpose of having a clear picture of the effect of each variant on the structure of $R R M 2 B$, we need the three-dimensional structure $(3 \mathrm{D})$ of native protein and its different mutated forms. This was done by homology modeling using the SWISS-MODEL server [30, 31].
TABLE 3: Number of conserved residues in the RRM2B protein provided by the SCR prediction server.

\begin{tabular}{lc}
\hline Sequence length & 351 \\
Structural conservation cutoff & 0.74 \\
Residues predicted to be conserved & 225 \\
\hline
\end{tabular}

2.5. Sequence Visualization and Interaction Change Observation. After obtaining the PDB files of the 3D models, YASARA software [32] was used to visualize and evaluate possible changes in the interactions between amino acids comparing to the native model, using the various features and tools available.

\section{Results}

3.1. SNP's Distribution. During this study, we managed to extract a total of 8464 SNPs of the RRM2B gene using the dbSNP database of NCBI. 6621 of these SNPs are in the intron region, 720 in the $3^{\prime}$ UTR region, 552 upstream, 184 nonsynonymous, 125 in the $5^{\prime}$ UTR region, 114 downstream, 74 synonymous, and 74 others (Figure 1). This study focuses only on nonsynonymous SNPs.

3.2. Deleterious nsSNPs of the RRM2B Gene. The results of the 13 prediction algorithms showed that 19 among 184 nsSNPs were deleterious (Table 1). For the majority of prediction software, 1 is the higher score and 0 is the lower score, except for the SIFT tool where the higher score is 0 and the lower score is 1 . 


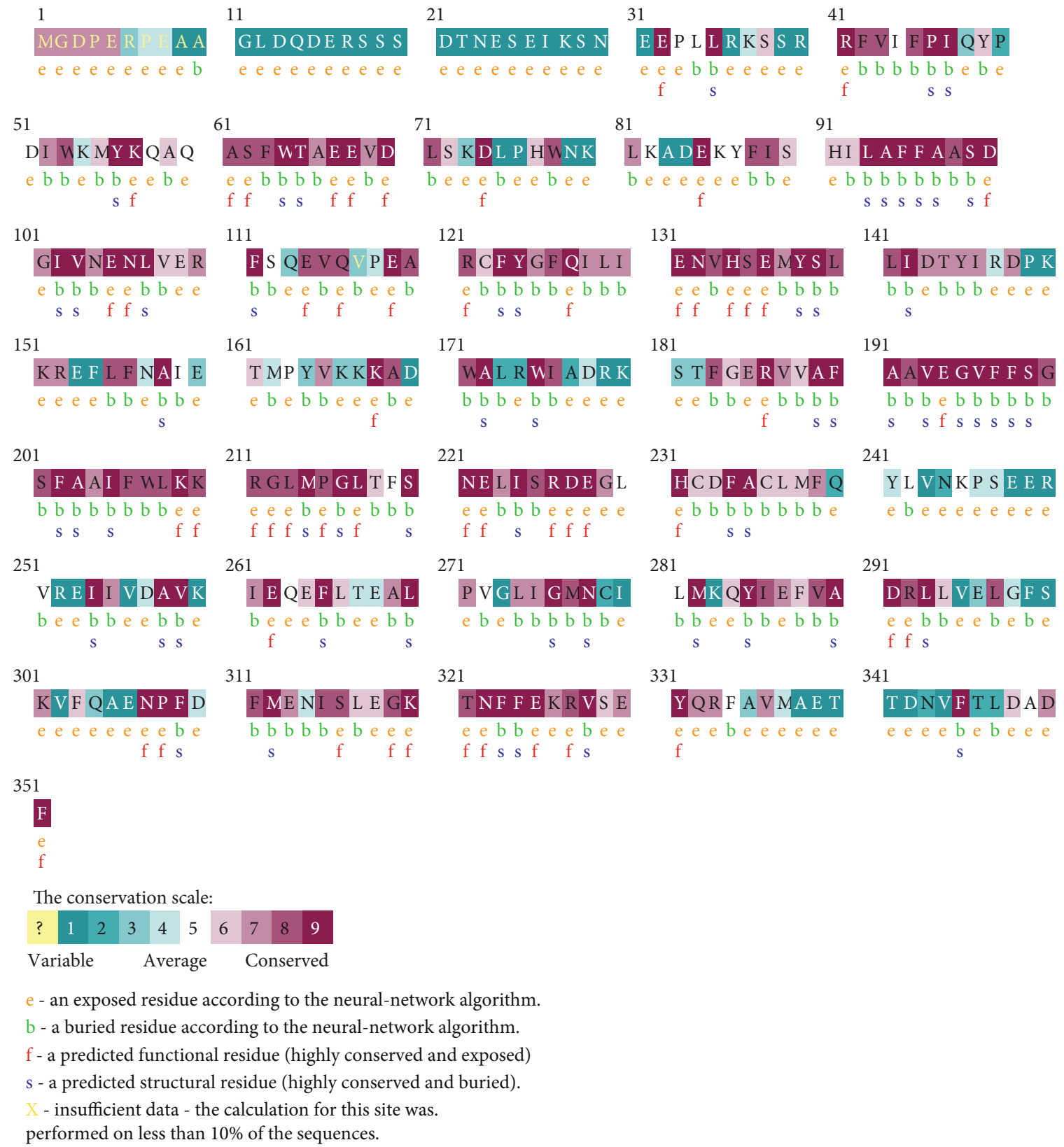

FIgURE 2: Conservation results of the RRM2B protein provided by the ConSurf web server.

3.3. Stability Analysis. To investigate the effect of each of the 19 nsSNPs on the stability of the RRM2B protein, two web tools were used: I-Mutant and Mupro. According to the DDG values, only $1 \mathrm{nsSNP}$ (Y124C) increased the stability of the RRM $2 B$ protein according to I-Mutant prediction, while for Mupro, all the 19 nsSNPs decreased the protein stability (Table 2).

3.4. Conservation Analysis. According to the conservation scale color code, we can see that the RRM2B protein sequence contains a high proportion of conserved regions, which make this protein more vulnerable for structural property alterations. These observations were confirmed through the examination of the exact number of conserved residues upon the $R R M 2 B$ protein sequence, using the SCR prediction web server. The results showed that the $R R M 2 B$ protein contains a total of 225 (out of 351) conserved residue representing $64 \%$ of the whole protein sequence (Table 3 ).

Using the ConSurf server, all the $19 \mathrm{nsSNP}$ residues are predicted to be located in conserved regions of the $R R M 2 B$ protein, representing exposed or buried and/or structural or functional residues. It is to mention that a structural residue is an amino acid that provides a structural framework to the protein, while a functional residue is an amino acid that mediates interactions of the protein with other biomolecules.

According to the conservation results, 16 nsSNPs were found to be localized in very highly conserved regions ( 9 on the conservation scale); 9 residues were predicted to be both exposed and functional, whereas 7 amino acids were buried and structural. 


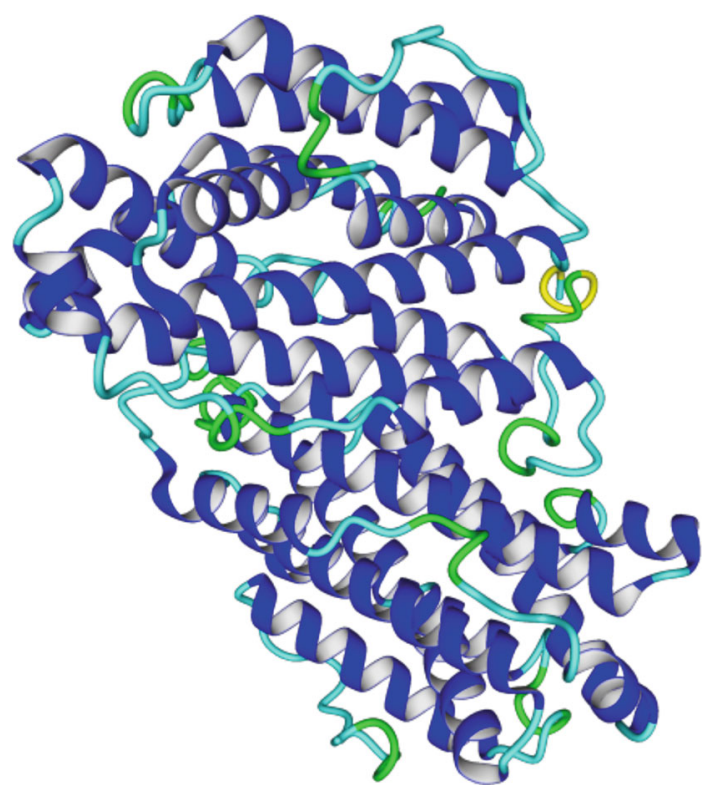

FIGURE 3: The RRM2B protein structure predicted using the SWISSMODEL server.

Two buried nsSNPs were located in a conserved region ( 8 on the conservation scale). The remaining SNP was found in a moderately conserved region ( 7 on the conservation scale) (Figure 2).

3.5. Molecular Modeling. The SWISS-MODEL server generated $3 \mathrm{D}$ structures of the wild-type and mutated proteins based on the 4DJN template. This template was the perfect candidate for the homology modeling, thanks to its high resolution of $2.2 \AA$ and other important variables (Qmean, coverage and sequence identity). Protein PDB files were downloaded from this web server and visualized using the YASARA software (Figure 3).

3.6. Structural Analysis. After visualizing the structure of the wild-type and mutated proteins using YASARA, we have noticed several differences in the interactions between amino acids (hydrogen or hydrophobic bonds).

As illustrated in Figure 4(a), the Arg amino acid in position 41 of the native form of $R R M 2 B$ protein that has a hydrophobic bond with Gln 48 and hydrogen bonds with Ile 44 and Glu 119 lost interactions after being replaced by Pro (Figure 4(b)) or by Trp (Figure 4(c)).

In the wild-type protein, the Phe 123 residue kept his 2 hydrogen bonds with Gln 127 and Glu 119 (Figure 5(a)) and his hydrophobic bond with Phe 234 when replaced by Ser (Figure 5(b)). Amino acid interaction analysis for all studied nsSNPs is summarized in Table 4, and their corresponding illustrations can be found in the supplementary materials.

Mutated proteins were superimposed with the wild type to calculate the root-mean-square deviation (RMSD). For all structures, the RMSD $(\AA)$ values did not deviate significantly from the native protein (Table 5 ).

\section{Discussion}

Pathogenic variants affecting $R R M 2 B$ protein structural properties can lead to remarkable mtDNA disruption whether on the qualitative (accumulation of multiple mtDNA deletions) or quantitative (depletion of mtDNA copy number) level, causing the appearance of serious phenotypes.

In order to understand the molecular origin of these phenotypes, experimental validation seems to be crucial, although these methods require both time and resource investment. In this regard, computational tools may be an intelligent way to do this kind of studies, through the easy and open access to different software and servers dedicated to the extraction of useful information about the impact of mutations, these tools can orientate the experimental studies in the future to be more efficient and precise. Through this study, we attempted to investigate the effect of deleterious nsSNPs on the structure of the RRM $2 B$ protein, via different bioinformatic tools. After using a total of 13 algorithms, only nsSNPs jugged deleterious were taken into consideration allowing us to move from 8464 SNPs to 19 deleterious nsSNPs: eight SNPs in exon 6 (R186G, E194G, E194K, G195R, G200E, F206V, G216R, and D227V), 7 in exon 4 (R110P, F123S, Y124C, E131A, E131K, S139R, and I142T), 2 in exon 7 (H231Q and A235T), and 2 in exon 2 (R41W and R41P).

A stability analysis was then conducted showing that only 1 nsSNP (Y124C) increased the stability of the RRM2B protein according to I-Mutant prediction, while for the Mupro tool, all the 19 nsSNPs decreased the stability. For the conservation analysis, the results have shown that the RRM2B protein is predicted to have a total of 225 conserved residues and that 16 out of the analyzed nsSNPs are localized in very highly conserved regions; 9 SNPs represent exposed and functional residues, while 7 SNPs represent buried and structural residues. These results may lead us to suspect that most of these variants may cause important alterations of the RRM2B protein.

Our results have shown that the majority of variants are responsible for important impacts on the protein structure and amino acid interactions. Only one nsSNPs (F123S) had no impact on the amino acid interactions; these results cannot exclude the fact that this variant may have an important effect on the protein structure, since it was predicted to be deleterious, stability decreasing, and located in a highly conserved region.

A variety of experimental trials concerning the $R R M 2 B$ gene have shown that some of the 19 nsSNPs concerned by this study are responsible for serious manifestations within subjects of various ages and phenotypes. Mutation p.Arg41Trp was found in a patient with PEO, ptosis, migraine, neck flexion weakness (mild), and proximal muscle weakness, the age of onset was 60 , and the patient died at the age of 66. While the heterozygous mutation p.Arg186Gly was found along with the p.Thr218Ile mutation in a 43-year-old patient with Severe PEO, asymmetrical ptosis, proximal and distal muscle weakness, ataxia, SNHL (sensorineural hearing loss), facial weakness, low BMI (Body Mass Index), leukoencephalopathy, and depression, with an age of onset of 11 


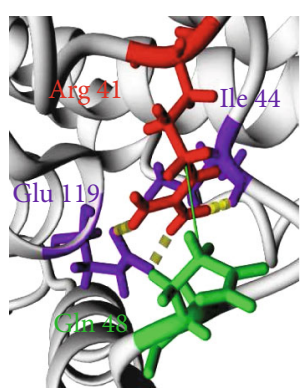

(a)

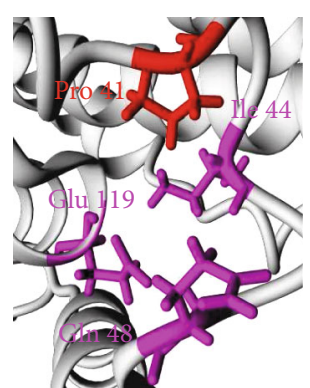

(b)

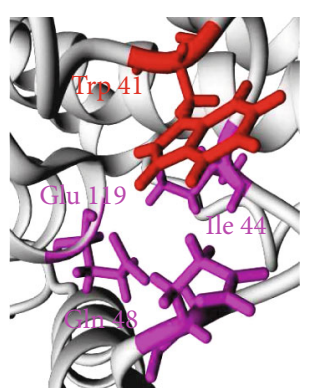

(c)

FIgURE 4: Comparison of the native RRM2B protein structure and two mutant forms. (a) The structural model of the wild-type protein (Arg 41). (b) The structural model of the first mutated form (Pro 41). (c) The structural model of the second mutated form (Trp 41). Discontinuous cylinders represent hydrogen bonds; continuous lines represent hydrophobic bonds. Red residues are the main residues where the nsSNPs appeared; purple residues are those who that have a hydrogen bond with the main residue; green residues are those who that have a hydrophobic bond with the main residue; magenta residues mark the loss of a bond between an amino acid and the main residue in the variant form that existed in the wild-type form.

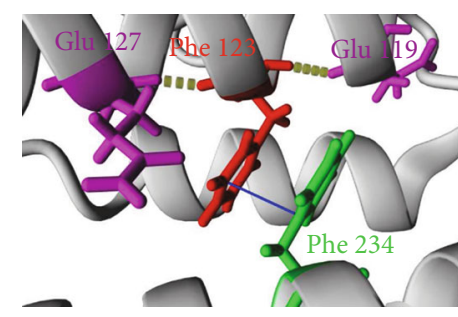

(a)

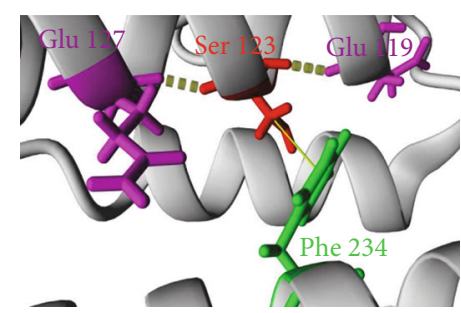

(b)

FIGURE 5: Comparison of the native RRM2B protein structure and a mutant form. (a) The structural model of the wild-type protein (Phe 123). (b) The structural model of the mutated form (Ser 123).

years old, another patient with Severe PEO, ptosis, SNHL, dysphonia, proximal muscle weakness, ataxia, cataracts, glaucoma, and IHD (ischemic heart disease) had the p.Gly195Arg mutation, with an age of onset of 50 years old [15].

On the other hand, Acham-Roschitz et al. reported a case of a baby girl of 8 weeks that suffered from muscular hypotonia with lack of head control as well as congenital deafness, nephrocalcinosis, and Fanconi syndrome. The genetic analysis revealed the presence of the p.Phe123Ser mutation [7]. The p.Glu194Lys mutation was reported in two siblings, a boy that showed trunk hypotonia and tubulopathy shortly after birth and died at the age of 2 months after status epilepticus and his sister that died at the same age and showed neonatal hyperlactatemia in addition to some similar complications as her brother. This mutation was also found in a baby girl that showed hypotonia soon after birth and developed respiratory distress, hyperlactatemia, and tubulopathy at an age of 3 months then died at the age of 4 months [5]. Finally, according to the study of Pitceathly et al., the p.Glu131Lys mutation was found along with p.Arg41Gln mutation in a patient with KSS syndrome [13].

Many studies are aimed at explaining the mechanism of these diseases as well as the functional consequence of their corresponding variants. Actually, the p.Arg41Trp mutation is predicted to prevent salt bridge formation. This bridge has an important role in conformation change that controls iron binding; this prediction was based on previous molecular modeling studies showing that p.Arg41Gln prevents salt bridge formation $[13,33]$.

As for mutations in the Arg 186 residue, it is demonstrated that they might be responsible for protein folding efficiency reduction leading to autosomal recessive disease, due to the presence of the residue at the end of the $\alpha$-helix [15].

Likewise, Gly 195 substitution impacts some of its neighboring amino acids that contribute to iron atom coordination; this disruption is associated with adult-onset chronic progressive external ophthalmoplegia (CPEO)/multiple mtDNA deletion disorders [13].

All this experimental evidence correlates strongly with the results obtained from the bioinformatic study that we have conducted, thus proving and explaining the effect of deleterious nsSNPs on the $R R M 2 B$ gene.

\section{Conclusion}

In the current study, we identified 19 nonsynonymous single nucleotide polymorphisms based on different computational prediction tools. To investigate the structural impact of these nsSNPs, molecular modeling analyses were done. A potential impact on protein stability was observed in 18 mutated structures in comparison with native protein. In addition, 18 variants may affect hydrogen bond and hydrophobic 


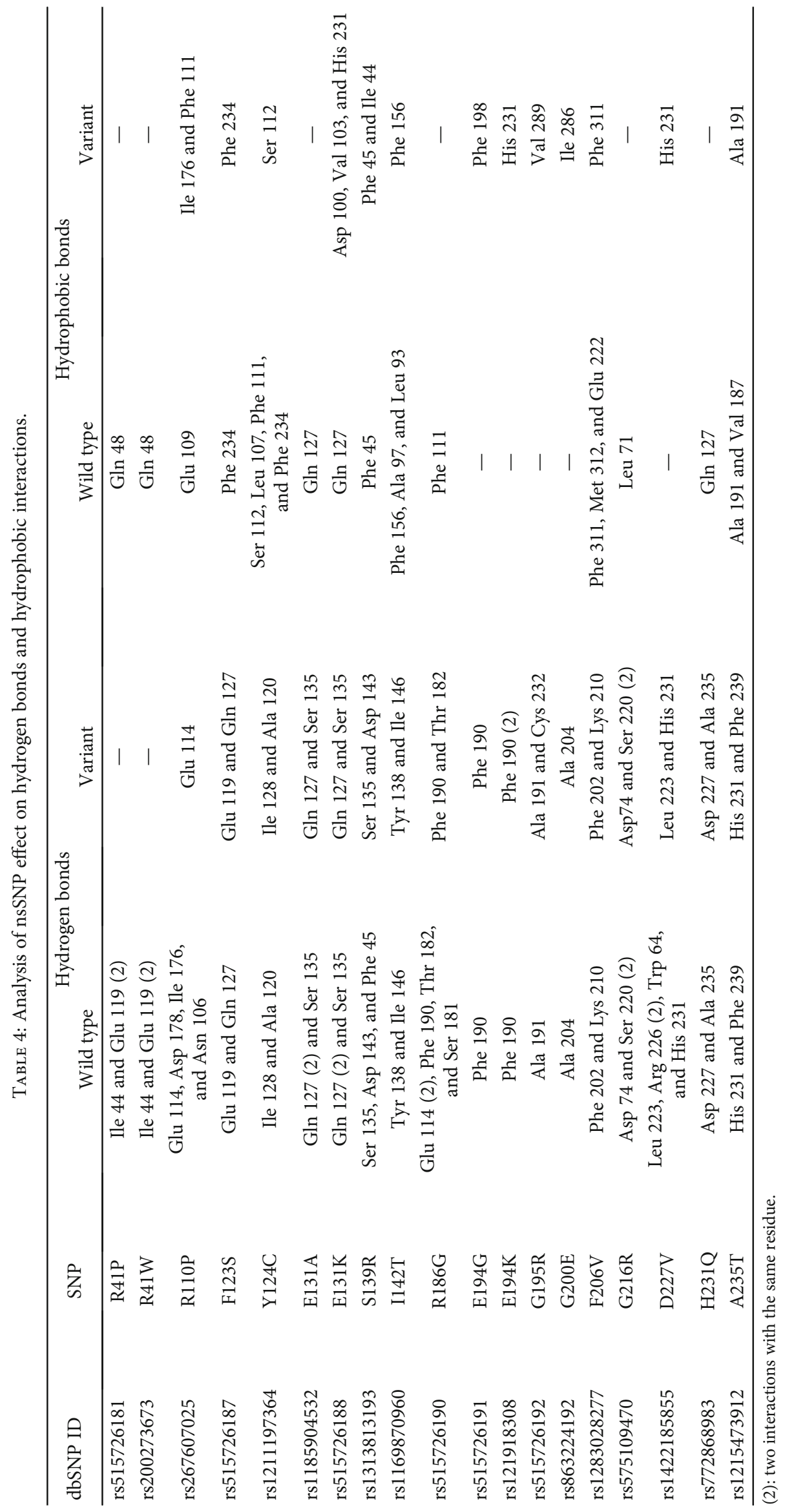


TABle 5: Root-mean-square deviation (RMSD) of deleterious nsSNPs.

\begin{tabular}{lcc}
\hline dbSNP ID & SNP & RMSD $(\AA)$ \\
\hline rs515726181 & R41W & 0.0274 \\
rs200273673 & R41P & 0.0236 \\
rs267607025 & R110P & 0.0446 \\
rs515726187 & F123S & 0.0032 \\
rs1211197364 & Y124C & 0.0019 \\
rs1185904532 & E131A & 0.0165 \\
rs515726188 & E131K & 0.0275 \\
rs1313813193 & S139R & 0.0964 \\
rs1169870960 & I142T & 0.0025 \\
rs515726190 & R186G & 0.0114 \\
rs515726191 & E194G & 0.0160 \\
rs121918308 & E194K & 0.0239 \\
rs515726192 & G195R & 0.0378 \\
rs863224192 & G200E & 0.0537 \\
rs1283028277 & F206V & 0.0059 \\
rs575109470 & G216R & 0.0684 \\
rs1422185855 & D227V & 0.0101 \\
rs772868983 & H231Q & 0.0181 \\
rs1215473912 & A235T & 0.0251 \\
\hline
\end{tabular}

interactions. Our results allow for a better understanding of the effect of RRM2B gene nsSNPs. Therefore, these SNPs should be considered important candidates in the genetic screening of neurological diseases, such as PEO and encephalomyopathy.

\section{Data Availability}

All data used in this study are included within the article and supplementary information file.

\section{Conflicts of Interest}

The authors declare that they have no conflicts of interest.

\section{Acknowledgments}

The authors are thankful to Pasteur Institute of Morocco for providing encouragement and facilities.

\section{Supplementary Materials}

Figure S1: comparison of the native RRM2B protein structure and a mutant form. (A) The structural model of the wild-type protein (Arg 110). (B) The structural model of the mutated form (Pro 110). Figure S2: comparison of the native RRM2B protein structure and a mutant form. (A) The structural model of the wild-type protein (Tyr 124). (B) The structural model of the mutated form (Cys 124). Figure S3: comparison of the native RRM2B protein structure and two mutant forms. (A) The structural model of the wild-type protein (Glu 131). (B) The structural model of the first mutated form (Ala 131). (C) The structural model of the second mutated form (Lys 131). Figure S4: comparison of the native RRM2B protein structure and a mutant form. (A) The structural model of the wild-type protein (Ser 139). (B) The structural model of the mutated form (Arg 139). Figure S5: comparison of the native RRM2B protein structure and a mutant form. (A) The structural model of the wild-type protein (Ile 142). (B) The structural model of the mutated form (Thr 142). Figure S6: comparison of the native RRM2B protein structure and a mutant form. (A) The structural model of the wild-type protein (Arg 186). (B) The structural model of the mutated form (Gly 186). Figure S7: comparison of the native RRM2B protein structure and two mutant forms. (A) The structural model of the wild-type protein (Glu 194). (B) The structural model of the first mutated form (Gly 194). (C) The structural model of the second mutated form (Lys 194). Figure S8: comparison of the native RRM2B protein structure and a mutant form. (A) The structural model of the wild-type protein (Gly 195). (B) The structural model of the mutated form (Arg 195). Figure S9: comparison of the native RRM2B protein structure and a mutant form. (A) The structural model of the wild-type protein (Gly 200). (B) The structural model of the mutated form (Glu 200). Figure S10: comparison of the native RRM2B protein structure and a mutant form. (A) The structural model of the wild-type protein (Phe 206). (B) The structural model of the mutated form (Val206). Figure S11: comparison of the native RRM2B protein structure and a mutant form. (A) The structural model of the wild-type protein (Gly 216). (B) The structural model of the mutated form (Arg 216). Figure S12: comparison of the native RRM2B protein structure and a mutant form. (A) The structural model of the wild-type protein (Asp 227). (B) The structural model of the mutated form (Val 227). Figure S13: comparison of the native RRM2B protein structure and a mutant form. (A) The structural model of the wild-type protein (His 231). (B) The structural model of the mutated form (Gln 231). Figure S14: comparison of the native RRM2B protein structure and a mutant form. (A) The structural model of the wild-type protein (Ala 235). (B) The structural model of the mutated form (Thr 235). (Supplementary Materials)

\section{References}

[1] D. C. Wallace, "Mitochondrial diseases in man and mouse," Science, vol. 283, no. 5407, pp. 1482-1488, 1999.

[2] L. Thelander and P. Reichard, "Reduction of ribonucleotides," Annual Review of Biochemistry, vol. 48, no. 1, pp. 133-158, 1979.

[3] P. Reichard, "Ribonucleotide reductases: the evolution of allosteric regulation," Archives of Biochemistry and Biophysics, vol. 397, no. 2, pp. 149-155, 2002.

[4] G. Nocentini, "Ribonucleotide reductase inhibitors: new strategies for cancer chemotherapy," Critical Reviews in Oncology/Hematology, vol. 22, no. 2, pp. 89-126, 1996.

[5] A. Bourdon, L. Minai, V. Serre et al., "Mutation of RRM2B, encoding p53-controlled ribonucleotide reductase (p53R2), causes severe mitochondrial DNA depletion," Nature Genetics, vol. 39, no. 6, pp. 776-780, 2007.

[6] M. H. Bornstein, C. S. Tamis-LeMonda, C.-S. Hahn, and O. M. Haynes, "Maternal responsiveness to young children at three 
ages: longitudinal analysis of a multidimensional, modular, and specific parenting construct," Developmental Psychology, vol. 44, no. 3, pp. 867-874, 2008.

[7] B. Acham-Roschitz, B. Plecko, F. Lindbichler et al., "A novel mutation of the RRM2B gene in an infant with early fatal encephalomyopathy, central hypomyelination, and tubulopathy," Molecular Genetics and Metabolism, vol. 98, no. 3, pp. 300-304, 2009.

[8] G. Kollberg, N. Darin, K. Benan et al., "A novel homozygous RRM2B missense mutation in association with severe mtDNA depletion," Neuromuscular Disorders, vol. 19, no. 2, pp. 147150, 2009.

[9] A. Shaibani, O. A. Shchelochkov, S. Zhang et al., "Mitochondrial neurogastrointestinal encephalopathy due to mutations in RRM2B," Archives of Neurology, vol. 66, no. 8, pp. 10281032, 2009.

[10] A. Spinazzola, F. Invernizzi, F. Carrara et al., "Clinical and molecular features of mitochondrial DNA depletion syndromes," Journal of Inherited Metabolic Disease, vol. 32, no. 2, pp. 143-158, 2009.

[11] H. Tyynismaa, E. Ylikallio, M. Patel, M. J. Molnar, R. G. Haller, and A. Suomalainen, "A heterozygous truncating mutation in RRM2B causes autosomal-dominant progressive external ophthalmoplegia with multiple mtDNA deletions," American Journal of Human Genetics, vol. 85, no. 2, pp. 290-295, 2009.

[12] C. Fratter, P. Raman, C. L. Alston et al., "RRM2B mutations are frequent in familial PEO with multiple mtDNA deletions," Neurology, vol. 76, no. 23, pp. 2032-2034, 2011.

[13] R. D. S. Pitceathly, E. Fassone, J.-W. Taanman et al., "KearnsSayre syndrome caused by defective R1/p53R2 assembly," Journal of Medical Genetics, vol. 48, no. 9, pp. 610-617, 2011.

[14] A. Takata, M. Kato, M. Nakamura et al., "Exome sequencing identifies a novel missense variant in RRM2B associated with autosomal recessive progressive external ophthalmoplegia," Genome Biology, vol. 12, no. 9, p. R92, 2011.

[15] R. D. S. Pitceathly, C. Smith, C. Fratter et al., "Adults with RRM2B-related mitochondrial disease have distinct clinical and molecular characteristics," Brain, vol. 135, no. 11, pp. 3392-3403, 2012.

[16] N.-L. Sim, P. Kumar, J. Hu, S. Henikoff, G. Schneider, and P. C. $\mathrm{Ng}$, "SIFT web server: predicting effects of amino acid substitutions on proteins," Nucleic Acids Research, vol. 40, no. W1, pp. W452-W457, 2012.

[17] I. Adzhubei, D. M. Jordan, and S. R. Sunyaev, "Predicting functional effect of human missense mutations using PolyPhen-2," Current Protocols in Human Genetics, vol. 76, no. 1, pp. 7.20.17.20.41, 2013.

[18] A. D. Johnson, R. E. Handsaker, S. L. Pulit, M. M. Nizzari, C. J. O'Donnell, and P. I. W. de Bakker, "SNAP: a web-based tool for identification and annotation of proxy SNPs using HapMap," Bioinformatics, vol. 24, no. 24, pp. 2938-2939, 2008.

[19] E. Capriotti, R. Calabrese, and R. Casadio, "Predicting the insurgence of human genetic diseases associated to single point protein mutations with support vector machines and evolutionary information," Bioinformatics, vol. 22, no. 22, pp. 2729-2734, 2006.

[20] A. González-Pérez and N. López-Bigas, "Improving the assessment of the outcome of nonsynonymous SNVs with a consensus deleteriousness score, condel," The American Journal of Human Genetics, vol. 88, no. 4, pp. 440-449, 2011.
[21] Y. Choi, G. E. Sims, S. Murphy, J. R. Miller, and A. P. Chan, "Predicting the functional effect of amino acid substitutions and indels," PLoS One, vol. 7, no. 10, p. e46688, 2012.

[22] K. A. Jagadeesh, A. M. Wenger, M. J. Berger et al., "M-CAP eliminates a majority of variants of uncertain significance in clinical exomes at high sensitivity," Nature Genetics, vol. 48, no. 12, pp. 1581-1586, 2016.

[23] S. Chun and J. C. Fay, "Identification of deleterious mutations within three human genomes," Genome Research, vol. 19, no. 9, pp. 1553-1561, 2009.

[24] B. Reva, Y. Antipin, and C. Sander, "Predicting the functional impact of protein mutations: application to cancer genomics," Nucleic Acids Research, vol. 39, no. 17, article e118, 2011.

[25] J. M. Schwarz, D. N. Cooper, M. Schuelke, and D. Seelow, "MutationTaster2: mutation prediction for the deepsequencing age," Nature Methods, vol. 11, no. 4, pp. 361-362, 2014.

[26] J. Bendl, J. Stourac, O. Salanda et al., "PredictSNP: robust and accurate consensus classifier for prediction of disease-related mutations," PLoS Computational Biology, vol. 10, no. 1, article e1003440, 2014.

[27] E. A. Stone and A. Sidow, "Physicochemical constraint violation by missense substitutions mediates impairment of protein function and disease severity," Genome Research, vol. 15, no. 7, pp. 978-986, 2005.

[28] H. Mi, B. Lazareva-Ulitsky, R. Loo et al., "The PANTHER database of protein families, subfamilies, functions and pathways," Nucleic Acids Research, vol. 33, pp. D284-D288, 2004.

[29] J. F. Borgio, M. S. Al-Madan, and S. AbdulAzeez, "Mutation near the binding interfaces at $\alpha$-hemoglobin stabilizing protein is highly pathogenic," American Journal of Translational Research, vol. 8, no. 10, pp. 4224-4232, 2016.

[30] S. Bienert, A. Waterhouse, T. A. P. de Beer et al., "The SWISSMODEL repository-new features and functionality," Nucleic Acids Research, vol. 45, no. D1, pp. D313-D319, 2017.

[31] A. Waterhouse, M. Bertoni, S. Bienert et al., "SWISS-MODEL: homology modelling of protein structures and complexes," Nucleic Acids Research, vol. 46, no. W1, pp. W296-W303, 2018.

[32] E. Krieger and G. Vriend, "YASARA view - molecular graphics for all devices - from smartphones to workstations," Bioinformatics, vol. 30, no. 20, pp. 2981-2982, 2014.

[33] P. Smith, B. Zhou, N. Ho et al., "2.6 Å X-ray crystal structure of human p53R2, a p 53- inducible ribonucleotide reductase," Biochemistry, vol. 48, no. 46, pp. 11134-11141, 2009. 